\title{
Modelling of Tsunami Due to Submarine Landslide by Smoothed Particle Hydrodynamics Method
}

\author{
Vo Nguyen Phu Huan ${ }^{1 *}$, Indra Sati H. Harahap ${ }^{2}$, and Wesam Salah Alaloul2 ${ }^{*}$ \\ ${ }^{1}$ Civil Engineering Faculty, The Open University of Ho Chi Minh City, Vietnam \\ ${ }^{2}$ Department of Civil and Environmental Engineering, University Technology PETRONAS, Perak, 32610, Malaysia
}

\begin{abstract}
Submarine landslide is the most serious threat on both local and regional scales. By way of addition to destroying directly offshore structures, slope failures may also generate destructive tsunami waves. This study has developed a numerical model based on the Smoothed Particle Hydrodynamics (SPH) method to predict four stages of generation, propagation, run-up, and impact of tsunami phenomenon. The numerical predictions in the research were validated with results in the literature and experimental tests. The results of the physical and numerical results presented in this study effort to develop these rule of thumbs to clearly understand some of the mechanics that may play a role in the assessment of tsunami waves.
\end{abstract}

\section{Introduction}

Tsunamis are surface waves generated by the impulsive disturbance under sea. Apart from co-seismic sea bottom displacement due to earthquakes, volcanic eructation, and submarine landslide can also create extreme tsunami waves with large and complex wave run-up. Presently, the physical understanding of this hazard is very poor. Therefore, this informs an immediate need for research to understand its dynamics, so that devastating events can be mitigated like Papua New Guinea, Indian Ocean or Brunei Slide's with potential impacts on affected coastal areas, on particular reference to East Malaysia and Brunei Darussalam. This is, of course, not a simple task because of the complexity and multi-scale of the process.

Until now, there is no effective numerical method can cover all stages of tsunami waves. To simulate and predict the tsunami is not an easy task, the present work has to face with many involved problems such as deformable borderline, free surface matter, huge deformation, time-running, costly task. With using NavierStokes Equations, the code based on the Smoothed Particle Hydrodynamics method, considered to be one of the most promising mesh-free methods, is due to the possibility of simulating generation, propagation, run-up, and impact of tsunami waves.

\section{Physical experiment}

Physical experiment was carried out to simplify the tsunami wave generation triggered by submarine slide, the experiments were conducted in narrow wave flume at Offshore Laboratory, Universiti Teknologi PETRONAS. Experimental test about tsunami wave was carried out in a concrete flume of $20 \mathrm{~m}$ length, $1.5 \mathrm{~m}$ width and $1 \mathrm{~m}$ depth. The side walls are composed of $1.28 \mathrm{~cm}$ thick tempered glass windows which were each $63.5 \mathrm{~cm}$ high and $1.52 \mathrm{~m}$ long. The bottom is constructed of high strength concrete material.

\footnotetext{
* Corresponding author : phuhuan129@yahoo.com, huan.vnp@ou.edu.vn, wesan.alaloul@petronas.com.my
} 

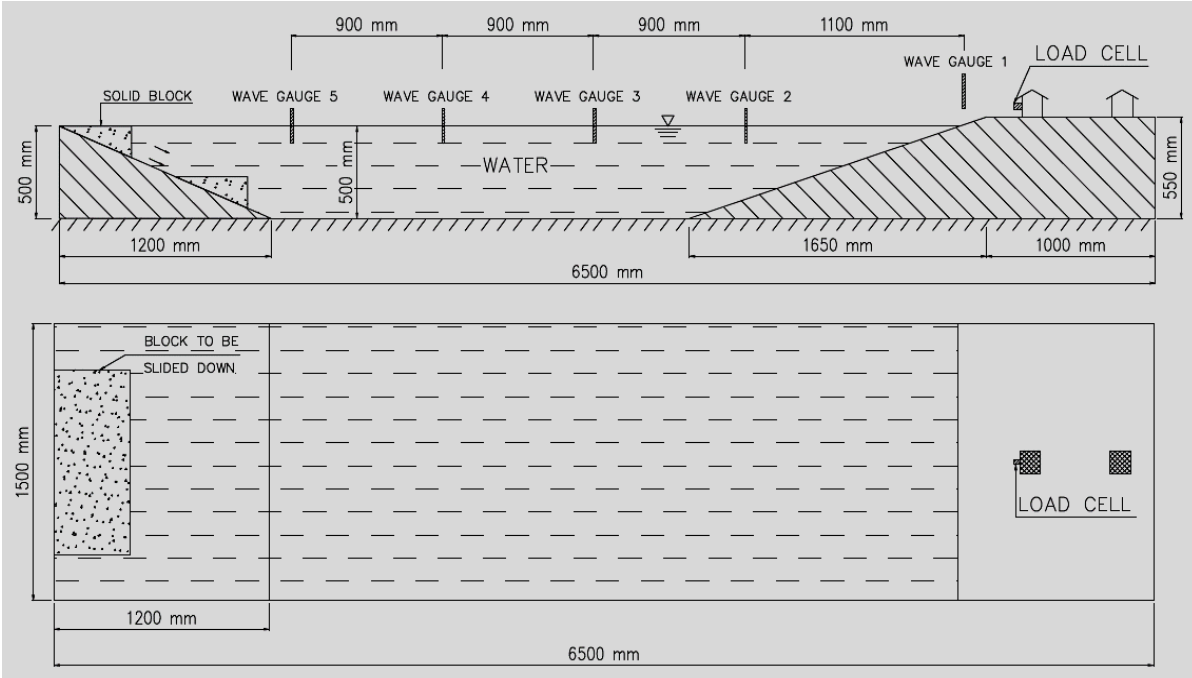

Fig. 1. Schematic diagram of experimental set-up for submarine slide generated tsunami waves

Fig. 1 shows the schematics of the experiment. The experimental setup included two inclined platforms. One of the inclined platforms was made for sliding down solid blocks, while th other for observation of the impact of slide-generated by tsunami waves. The sliding surface was smooth and was also lubricated in order to provide a frictionless slope. Tsunami waves were generated by sliding down solid blocks along the inclined bed. The blocks had a different shape, volume, and thickness and they have been made of steel plates with a rock inside. The total weight of block was determined based on the weight of steel plates, the weight of rock inside and the filling water weight. It was considered the block was full of water. With 5 wave gauges located at the central axis of the flume, the water elevation were measured exactly when wave propagation and run-up occur.

A 1:25 scale model of the houses $(100 \mathrm{~cm} \times 100 \mathrm{~cm} \times 100 \mathrm{~cm})$ was built using steel plates (Fig. 2). In addition, load cells were attached to the model houses and impact pressure will be assessed when tsunami wave hit the houses on the shore.

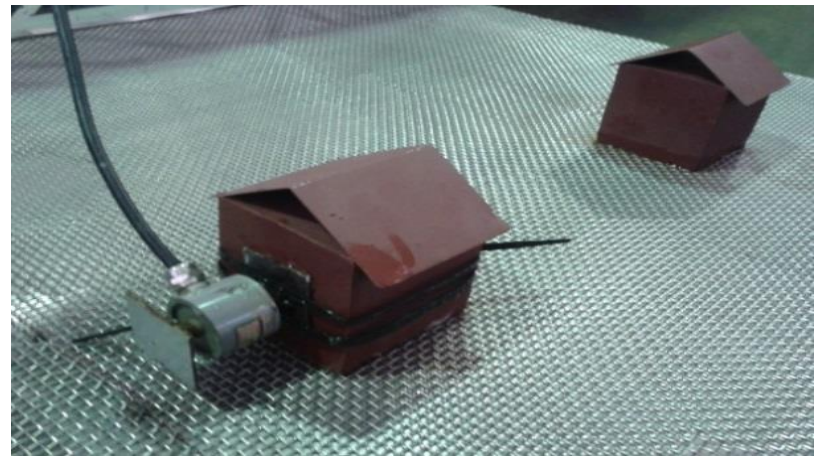

Fig. 2. Model houses were on the platform with the load cell.

\section{Numerical simulation}

These results from experimental test still cannot be used in real problem. The main purpose of these experiments is to both gain insight into landslide tsunami processes and provide data for subsequent validation of a numerical model. This brings into the picture the need for developing a numerical model in order to decrease the high costs associated with real large-scale physical experiments.

Monaghan, Lucy and Gingold (1977) were introduced Smooth Particle Hydrodynamics (SPH) as a new lagraian numerical model. The main advantages of SPH compared to Finite Difference Method (FDM) and Finite Volume Method (FVM) can be resume as follows: it is possible to emulate very huge phenomena in a relative easy way with no problem at the interface boundaries. In contrast with the FDM and FVM schemes, 
SPH is possible to create these open boundaries in an extremely easy way, this is important in particular in astrophysical application.

To reproduce the movement of a water and the interaction betwwen tsunami and model house, it is necessary to develop a SPH numerical model. However, the simulation will be sharply complicated as the number of particles in the SPH model increases. It is therefore necessary to parallelize the computing procedure without suffering from a loss of precision. The Message Passing Interface (MPI) parallelism for shared-memory programming enables loop-level parallelism.

Table 1. These most significant parameters were used in simulation

\begin{tabular}{|c|c|}
\hline Parameter & Value \\
\hline Particle Spacing (dx and dz) (m) & $0.001,0.001$ \\
\hline Smoothing Distance (h) (m) & 0.003 \\
\hline Smoothing Distance Coefficient & 0.027 \\
\hline Kernel Type & Cubic \\
\hline Density Filter & None \\
\hline Riemann Solver & Non-conservative \\
\hline $\begin{array}{c}\text { Riemann Solver`s Slope Limiter } \\
\text { (Beta-limiter) }\end{array}$ & 1.2 \\
\hline Reference Speed of Sound $(\mathrm{m} / \mathrm{s})$ & 32.21 \\
\hline Coefficient of Speed of Sound (B) & 15 \\
\hline Time Stepping Method & Simplistic \\
\hline Viscosity Treatment & Laminar + Sub-Particle Scale \\
\hline Viscosity term $(\alpha$ and $\beta)$ & 0.015 and 0 \\
\hline
\end{tabular}

The results of the numerical model should be compared to the data recorded in the physical experiments. Result in Fig. 3 shows that SPH code is an application of a unique comprehensive model that covers all aspects of slide induced tsunami from source generation to coastal impact.

Along the next frame (Fig. 3), different instants of the propagation, run-up and impact can be observed: $t=$ 0 s correspond to initial state of the simulation, $\mathrm{t}=1 \mathrm{~s}$ correspond to generate tsunami wave by sliding down solid block along on the inclined bed, $\mathrm{t}=2 \mathrm{~s}$ correspond to wave propagation and the tsunami run-up happen and hit house model at $\mathrm{t}=3 \mathrm{~s}$. 


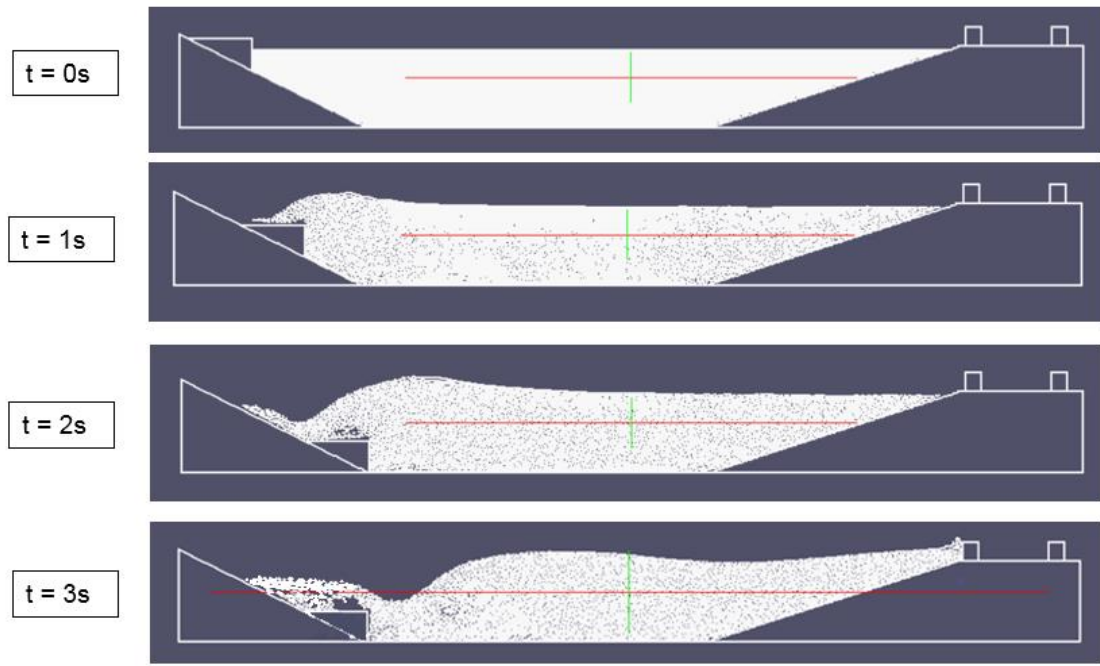

Fig. 3. Tsunami wave in a flume simulated with parallel SPHysics code 2D.

\section{Comparison}

The three frames below show both model and experimental results for surface elevation and impact pressure. The orange line and blue line represent the physical experiment and the SPH model respectively.

Readings at wave gauge (Fig.4) show that the numerical model wave height starts to peak around $2.0 \mathrm{~s}$ which is approximately at the same time as the physical experiment. The numerical signal is observed to have a slight difference with the experimental one, both in phase and in amplitude.

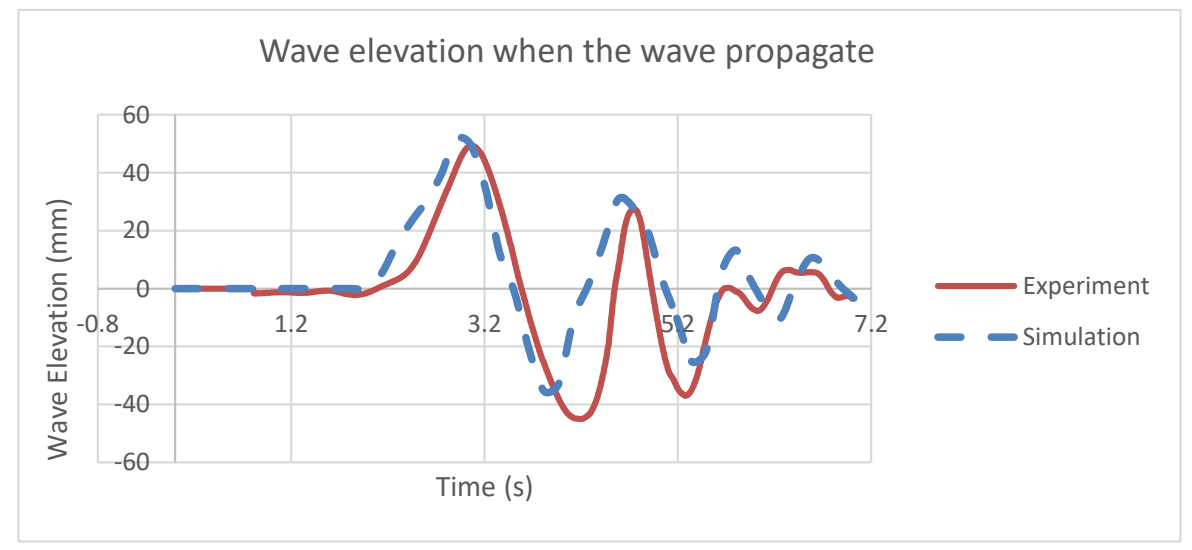

Fig.4. Comparison of tsunami propagation between numerical signal and experimental signal (propagation)

The wave is close to impact the house model and the peak wave height starts to occur around 3 s. (Fig. 5). The results show that near model house, free surface elevation (run-up) was more variable due to the complexity and variability of the wave breaking, wave reflection, and collapse of the water column in this zone. 


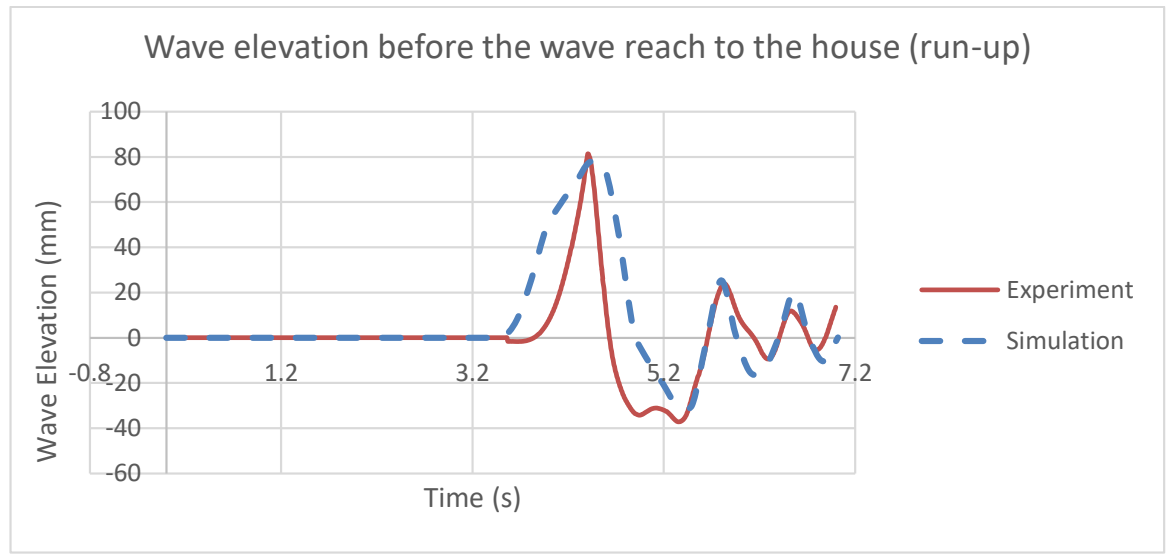

Fig. 5. Comparison of tsunami run-up between numerical signal and experimental signal (run-up)

Experimental and numerical data present some discontinuities and they were equally spaced $(\Delta t=0.015 \mathrm{~s})$. The series were considered in the interval to better capture the collision between the tsunami and the front of the house structure. As shown, for the load cell location, the numerical model results correctly depict the typical shape of the pressure-time history. Fig. 6 shows the slight difference between numerical and experimental pressures.

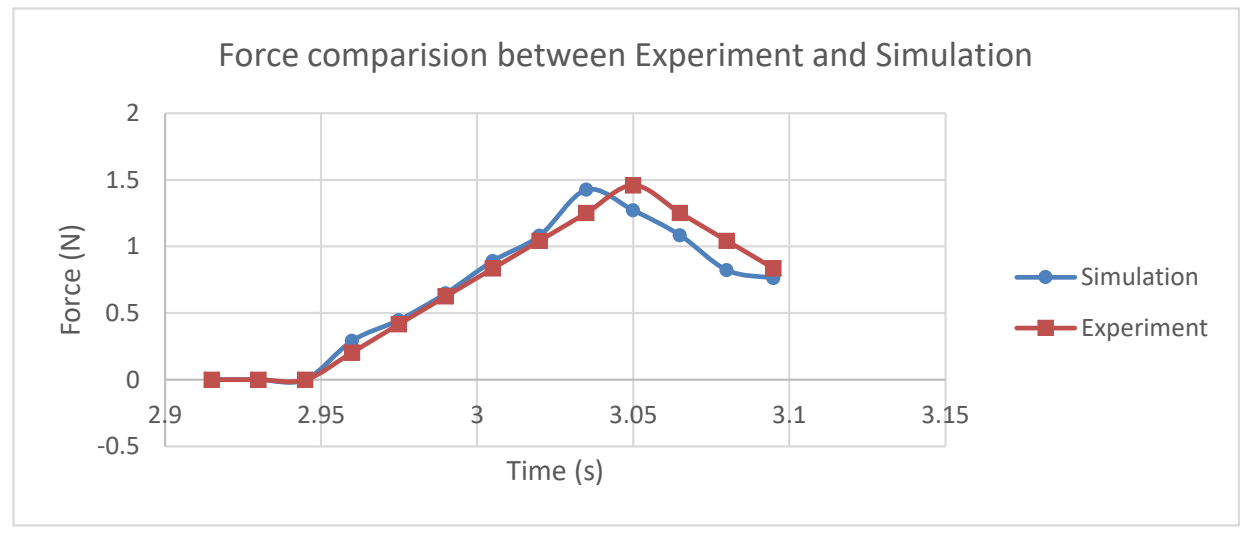

Fig. 6. Comparison of impact pressure on coastal house between numerical signal and experimental signal (Impact).

\section{Conclusions}

- To prevent the huge fluctuations in the pressure amplitude, a Riemann Solver was developed. The new SPH code in this research, the Riemann Solver is a parameter which support to reduce the level of disturbances during computation. The approach success due to estimate the impact pressure on coastal structures correctly.

- With using laminar SPS viscosity and adjust the coefficient of time stepping in new SPH code, the amount of instability were reduced effectively during the numerical simulation.

- The simulation of all stages of slide tsunami is a complex task because it requires a large of particles in SPH model. By using Open Message Passing Parallel libraries, this can be obtained assigning a parallel do loop cycle when updating the properties of particles in the fluid domain. Millions of particles could be simulated in this study, so the numerical results were more accurate and able to reduce the errors.

- The numerical SPH method can reasonably well model all stages of tsunami wave generated by submarine slide. It has been possible after that to have a better view on the evaluation of the viscous term in Navier-Stokes equation with SPH method.

- At present, efforts are under way for the elaboration of new design guidelines for tsunami-resistant structures. The results of the physical and numerical results presented in this thesis attempt to assist the 
developers of these guidelines to better understand some of the mechanisms that may play a role in the estimation of tsunami coastal flooding.

The authors would like to thank community members and experts, who generously contributed on this research. We ultimately hope they will benefit on prospective policy reform related to the study interest. The research is supported by the Ho Chi Minh City Open University (Project No. E2016.5.5.1) and the Universiti Teknologi PETRONAS

\section{References}

1. V. N. P. Huan, \& I. S. H. Harahap, Generation, Propagation, Run-up, and Impact of Landslide Triggered Tsunami: A Literature Review. Applied Mechanics and Materials, Vol. 567, 724-729 (2014)

2. V. N. P. Huan, \& I. S. H. Harahap, Computational Aspects of Submarine Slide Generated Tsunami. Applied Mechanics and Materials. Vol. 567, 216-221 (2014)

3. V. N. P. Huan, \& I. S. H. Harahap, Parallel Simulation of Tsunami Wave Generated by Submarine Slide: Generation, Propagation, Run-up and Impact. Applied Mechanics and Materials, Vol. 752-753, 1269-1274. (2015).

4. V. K. Gusiakov, Tsunami history: recorded in The Sea, in: Tsunamis, edited by: Robinson, A. and Bernard, E., Harvard University Press, Cambridge, 15, 23-54. (2009)

5. FEMA-P646 Guidelines for Design of Structures for Vertical Evacuation From Tsunamis. Washington, D.C., Federal Emergency Management Agency (2008)

6. E. Didier, and M. G. Neves, A Lagrangian Smoothed Particles Hydrodynamics - SPH - method for modelling waves-coastal structure interaction, 5th European Conference on Computational Fluid Dynamics, June 14-17, 2010, Lisbon, Portugal, (2010)

7. C. Altomare, Applicability of Smoothed Particle Hydrodynamics for estimation of sea wave impact on coastal structures. Coastal Engineering, 96, 1-12 (2015)

8. M. F. Chai, T. L. Lau, and T. A. Majid, Potential impacts of the Brunei Slide tsunami over East Malaysia and Brunei Darussalam. Ocean Engineering, 81, 69-76. (2014)

9. S. D. Chowdhury and S. A. Sannasiraj, SPH Simulation of Shallow Water Wave Propagation. Ocean Engineering 60, 41-52 (2013) 